\title{
Consensus statement on the management of invasive candidiasis in Indian scenario
}

\section{Prakash Shastri $^{1 *}$, Vivek Nangia ${ }^{2}$, Prachee Sathe ${ }^{3}$, Rajesh Pande ${ }^{4}$, Abi Abraham ${ }^{5}$, Ashit Hegde ${ }^{6}$, Abdul Samad Ansari ${ }^{7}$, Dharmesh Kapoor ${ }^{8}$, Amit Mandal', Alok Jain ${ }^{10}$, M. Dinaker ${ }^{11}$, Deepali Mohanty ${ }^{12}$, Dimbeshwar Das ${ }^{13}$, Venkat Ramana Kola ${ }^{14}$, Sharmili Sinha ${ }^{15}$, Amitav Mohanty ${ }^{16}$, Hardev Singh Girn ${ }^{17}$}

\author{
${ }^{1}$ Department of Critical Care and Emergency Medicine, Sir Ganga Ram Hospital, Delhi, India \\ ${ }^{2}$ Department of Pulmonology, Fortis Hospital, Noida, Uttar Pradesh, India \\ ${ }^{3}$ Department of Critical Care Medicine, Ruby Hall Clinic, Pune, Maharashtra, India \\ ${ }^{4}$ Department of Critical Care and Emergency Medicine, BLK Hospital, Delhi, India \\ ${ }^{5}$ Department of Nephrology and Renal Transplant services, Lakeshore Hospital, Kochi, Kerela, India \\ ${ }^{6}$ Department of Critical Care, PD Hinduja Hospital, Mumbai, Maharashtra, India \\ ${ }^{7}$ Department of Critical Care, Nanavati Super Speciality Hospital, Mumbai, Maharashtra, India \\ ${ }^{8}$ Department of Gastroenterology, Global Hospitals, India \\ ${ }^{9}$ Department of Critical Care, Pulmonology and Sleep Medicine, Fortis Healthcare, Punjab, India \\ ${ }^{10}$ Department of Nephrology, Rukmani Birla Hospital, Jaipur, Rajasthan, India \\ ${ }^{11}$ Department of Medicine, Sunshine Hospital, Hyderabad, India \\ ${ }^{12}$ Department of Medicine, Sahara Hospital, Lucknow, Uttar Pradesh India \\ ${ }^{13}$ Department of Medicine, ILS Hospital, Kolkata, India \\ ${ }^{14}$ Department of Critical Care, Care Hospital, Ahmedabad, India \\ ${ }^{15}$ Department of Critical Care, ${ }^{16}$ Department of Medicine and Critical Care, Apollo Hospital Bhubaneshwar, Odisha, \\ India \\ ${ }^{17}$ Department of Liver Transplant, Dayanand Medical College and Hospital, Punjab, India
}

Received: 22 August 2019

Revised: 26 September 2019

Accepted: 04 October 2019

\section{*Correspondence: \\ Dr. Prakash Shastri, \\ E-mail: prakashshastri@live.in}

Copyright: () the author(s), publisher and licensee Medip Academy. This is an open-access article distributed under the terms of the Creative Commons Attribution Non-Commercial License, which permits unrestricted non-commercial use, distribution, and reproduction in any medium, provided the original work is properly cited.

\section{ABSTRACT}

Invasive fungal infections in critically ill patients are associated with increased morbidity and mortality. Candida species are among the most common causes of nosocomial bloodstream infections and of invasive infections in intensive care units (ICUs). The high mortality mandates early identification of invasive candidiasis which is vital to initiate appropriate and timely treatment and improve outcomes. Delaying the initiation of treatment could result in an increase in mortality which can be avoided by usage of more rapid diagnostic techniques. There are multiple diagnostic tests including culture and non-culture tests like 1,3- $\beta$-D-glucan and newer techniques like MALDI-TOF which are available to diagnose candidemia but each with their drawbacks. Additionally, there are various guidelines like IDSA and ESCMID on treatment which aim to minimize death, late complications from deep-seated candidiasis and rise of drug- resistant Candida strains. Through this consensus statement prepared by a panel of experts, all of whom are senior intensivists, infectious disease specialists and microbiologists, we aim to address the major aspects of management of invasive candidiasis in the Indian population as per the authors opinions, backed by published evidence and supported by the latest clinical guidelines.

Keywords: Candida strains, Caspofungin, Invasive candidiasis, Non-culture tests, Matrix assisted laser desorption/ionization- time of flight 


\section{INTRODUCTION}

Invasive fungal infections have become a scourge in the intensive care units throughout the world. There has been an alarming rise in the incidence of invasive fungal infections, owing to disruption of natural barriers, complex medical and surgical problems, multiple invasive procedures and prolonged treatment with broad spectrum antibiotics. ${ }^{1}$

Amongst all the opportunistic infections in hospitals, infections with Candida species is the most common. ${ }^{2}$ They are the leading cause of catheter-associated blood stream infections in America and Europe and also one of the leading cause of ICU morbidity and mortality. By definition, the term 'invasive candidiasis' refers to a whole spectrum of diseases, not restricted only to candidemia. However, this may be due to the fact that the majority of research is limited to candidemia, attributable to the fact that diagnosing non-albicans candidiasis is not easy and hence goes undetected. ${ }^{3}$

There is a marked geographical variation in the species distribution of Candida. SENTRY antimicrobial surveillance program was designed to monitor the predominant pathogens and antimicrobial resistance for both nosocomial and community-acquired infections globally by using validated, reference-quality identification and susceptibility testing methods performed in a central laboratory. It evaluated a total of 2085 clinical Candida isolates, which were collected from 79 different medical centers across North America, Latin America, Europe and Asia. The most common species isolated from Asia-pacific region was Candida albicans (56.9\%) followed by Candida glabrata (13.7\%), Candida parapsilosis (13.7\%) and Candida tropicalis (11.7\%). North America had $43.4 \%$ of C. albicans, $23.5 \%$ of C. glabrata while Europe had $55 \%$ of C. albicans and $15.7 \%$ of C. glabrata. ${ }^{4}$

While the epidemiological data of Candida species in Indian scenario is scanty and sporadic, it does point toward the fact that the predominant species is $C$. tropicalis, instead of $C$. glabarata or $C$. parapsilosis. This rise in the incidence of NAC (non-albicans Candida) might be a fallout of increased use of fluconazole prophylaxis in immunocompromised patients, central venous cannulations and prior gastrointestinal surgery. ${ }^{2}$ Even the incidence of $C$. auris, which is a multidrug-resistant yeast associated with outbreaks in healthcare settings, is on the rise. Although the mortality rate attributable to $C$. auris is unknown, it is estimated to be around 30 to $60 \% .^{5}$ The incidence, as observed in a multicentric study conducted at 27 Indian ICUs, ranged from 4 to $8 \%$ of all candidemia infections. ${ }^{6}$ Thus, candidemia is a major issue facing the Indian ICUs today. This consensus statement is prepared by a panel of experts, all of whom are senior intensivists and microbiologists, and will aim to address the major aspects of management of invasive candidiasis as per the authors opinions, backed by published evidence and supported by the latest clinical guidelines. For the purpose of this document, the aspects related to management of invasive candidiasis in non-neutropenic ICU patients will be covered.

\section{DIAGNOSIS}

Early identification of invasive candidiasis is crucial to initiate appropriate and timely treatment and improve outcomes. It has been shown in a retrospective cohort study by Morrell et al, that delaying the initiation of treatment could result in an increase in mortality; indeed, this delay can be avoided by development and usage of more rapid diagnostic techniques for identifying Candida BSI. ${ }^{7}$ This is however easier said than done.

Tissue smearing and subsequent microscopic examination can be performed rapidly, however it has a very low negative predictive value. ${ }^{8}$

The gold standard for the diagnosis of candidemia is a positive blood culture; hence it is imperative to obtain blood cultures from all patients with suspected candidemia. ${ }^{9}$ They have good sensitivity in detecting viable Candida which is evident from the fact that the median Candida concentration is $1 \mathrm{CFU} / \mathrm{ml}$ at the time of the first positive blood culture. This limit of detection of viable Candida by blood cultures is reported to be as good as or superior as compared to that for methods such as PCR. The chances of getting a positive blood culture are greater if the samples are collected during active candidemia. ${ }^{10}$

However, the problems with blood cultures are numerous: They are positive in only $50 \%$ of patients with Candida blood stream infections; they have a slow turnaround time- one to three days are required for growth and an additional one to two days for identification of the organism after subculture onto agar; they are rarely positive in patients of deep-seated candidiasis; and finally collection of deep-tissue cultures requires invasive procedures that may be risky to the patient or even contraindicated in patients at-risk for Candida infections. ${ }^{8,10}$

Once the blood culture shows growth and subsequent microscopy reveals yeasts, newer techniques such as PNA FISH (peptide nucleic acid fluorescence in situ hybridization), some of the more common Candida species such as $C$. albicans and $C$. glabrata can be identified within a few hours of the culture becoming positive for yeasts. ${ }^{9}$

\section{Non-Culture Tests for Invasive Candidiasis}

Mannan, antimannan antibody, and C. albicans germ tube antibody (CAGTA)

Assays for Candida antigens and antibodies were some of the earliest serum assays developed. The major drawbacks of Candida antigens are that their serum levels are low and that they are rapidly cleared from the blood; 
while the major drawbacks of anti-Candida antibodies are that considerable time is needed to mount an immune response, they may not be reliable in immunosuppressed hosts due to very low production of antibodies, and that positive results may not be able to differentiate between acute and past infections. Despite these drawbacks mentioned above, they have been found to give good results when used in clinical practice. ${ }^{10}$ Rather than using mannan and anti-mannan tests individually, it is best to use them together as they have given good results. The CAGTA ( $C$. albicans germ tube antibody) test detects responses against a hyphal protein (Hwp1) expressed during tissue invasion and biofilm formation. ${ }^{10}$ CAGTA may be combined with $1,3-\beta$-D-glucan testing to improve diagnostic accuracy. ${ }^{3}$

\section{$B D G$}

$1,3-\beta$-D-glucan is a is a major cell wall constituent of Candida and most pathogenic fungi, excluding Cryptococcus species, Blastomyces species, and Mucorales. It is not species or genus- specific, rather just an indicator of invasive fungal infections; hence further tests are needed to identify the specific fungus. However, it can be very useful adjunct to blood cultures. For example, in intra-abdominal candidiasis, it can be invaluable in detecting deep-seated candidiasis when blood cultures are negative. This test has the drawback of false positivity in patients with risk factors such as Candida or mold colonization, human blood products, hemodialysis or hemofiltration, some gram positive bacteria, certain beta-lactam antibiotics, cellulose dressings, enteral nutrition, mucositis, and disruptions of GI tract integrity. ${ }^{9,10}$

\section{MALDI-TOF-MS}

The advent of MALDI-TOF-MS (matrix-assisted laser desorption ionization-time of flight mass spectrometry) as a standard diagnostic procedure for yeast identification may lead to a better recognition of rare Candida spp., such as $C$. auris, that were previously misdiagnosed or unrecognized. ${ }^{3}$ Proteins that are released from Candida species are detected and compared with a large database of proteins from many species of yeast. ${ }^{9}$ It gives results fairly quick and thus helps to significantly reduce the delay to initiate appropriate antifungal therapy based on the Candida species. ${ }^{11}$ It is performed on colonies that have grown from a blood culture bottle, and results can be read in as short a period as 30 minutes.

\section{T2Candida panel}

The T2Candida nano diagnostic Panel is approved by FDA for detecting candidemia and using an automated process detects Candida directly within whole blood. It lyses RBCs, concentrates Candida and then amplifies DNA. Results are reported as positive or negative for $C$. albicans/ C. tropicalis, C. glabrata/ C. krusei, and $C$. parapsilosis, groupings that are based on typical antifungal susceptibility patterns. ${ }^{10}$

\section{T2 magnetic resonance}

Another FDA approved technique is T2 magnetic resonance (which combines PCR and nanoparticle based hybridization) which is designed to detect the five most common species of Candida and has been reported to have $99.4 \%$ specificity for candidemia. This can help in direct detection of Candida spp. in blood samples may improve the early detection of IC. While automated blood cultures systems usually require 1-3 days for the detection of yeasts, T2MR can identify Candida spp. within several hours from the time of sampling. This test however is expensive and data on cost-benefit ratio does not exist to advocate its routine use. ${ }^{3,8}$

\section{PCR}

The polymerase chain reaction (PCR) assay may be a very useful non-culture method for the diagnosis of systemic fungal infection in high-risk patients and can identify the species of Candida. ${ }^{9}$ Data regarding use of PCR in critical care is limited and there is currently no approved test commercially available for detecting candidemia. ${ }^{1,9}$ However available evidence is encouraging. For the detection of candidemia, the sensitivity of PCR has been reported to be similar to that of blood cultures $(60 \%)$ and it has also proved to be effective in diagnosing deep-seated candidiasis with negative blood cultures. ${ }^{1}$

The molecular and serologic techniques for diagnosis can prove to be invaluable tools for the early diagnosis of IFI. Since the interpretation of positive or negative results with different laboratory methods is difficult for clinicians, therefore for accurate diagnosis, more than one of these methods should be used. ${ }^{12}$

\section{Risk Factor and scoring system- based methods}

There are several risk factors which have been identified for invasive Candida infections, including higher Acute Physiology and Chronic Health Evaluation (APACHE) II scores, diabetes mellitus, surgery (especially abdominal surgery), renal insufficiency, pancreatitis, the use of broad-spectrum antibiotics, parenteral nutrition, hemodialysis, mechanical ventilation, the presence of central vascular catheters, and therapy with immunosuppressive agents. Extensive colonization of the skin or of the mucus membranes of the gastrointestinal and urogenital tracts often precedes development of invasive Candida infections, and this degree of colonization, assessed using the Colonization Index, has been shown to be an independent risk factor for development of candidiasis.

Several prediction rules and scores have been proposed in recent years based on clinical, laboratory, and 
microbiological parameters. These are presumed to aid clinicians in identifying patients at high risk of developing invasive fungal infections. Ostrosky-Zeichner et al. proposed a prediction rule characterized by a very high negative predictive value $(0.97)$ and including the following parameters: use of systemic antibiotic therapy, total parenteral nutrition, dialysis, steroids or immunosuppressive agents, major surgery or pancreatitis, and the presence of a central venous catheter. Leon et al proposed the Candida score, an easy-to-use assessment system, integrates four risk factors (total parenteral nutrition, surgery, multifocal Candida colonization, and severe sepsis) and also has a high negative predictive value $(0.98)$ to rule out invasive candidiasis. A total score of 3 or more has $81 \%$ sensitivity and $74 \%$ specificity for the diagnosis of invasive candidiasis, and a 7.7-fold greater risk of candidiasis. $^{8}$

Pragmatically, the real value of risk scores and clinical rules lies not in their relatively low positive predictive value for diagnosing invasive candidiasis but their high negative predictive value for ruling out infection. This may allow clinicians to withhold otherwise unnecessary antifungal treatments in critically ill patients and thus help to aid in the minimization of the emergence of resistant fungal strains. ${ }^{11}$

\section{TREATMENT}

There are three primary objectives of the treatment of invasive candidiasis. It aims to minimize:

- Death, within 7 days of onset of infections;

- Late complications from deep-seated candidiasis;

- Rise of drug- resistant Candida strains. ${ }^{13}$

Indeed, it has been shown in a study by Morrell et al, that delaying the initiation of antifungal therapy in ICU patients is associated with greater chances of mortality. ${ }^{7}$

Prophylaxis strategy is defined as administration of antifungal agents to patients with risk factors for invasive candidiasis without clinical signs and symptoms of infection. Antifungal prophylaxis has been studied in ICU patients earlier; however, the quality of evidence is low and hence there is uncertainty with regards to outcomes and therefore optimal approach remains unclear, this expert panel does not recommend routine use of prophylaxis.

The best approach to the optimal management of invasive fungal infections involves early detection and identification of the invading organism, so that appropriate treatment can be initiated as soon as possible. Unfortunately, patients with fungal infection often die of complications attributed to the infection despite antifungal therapy. ${ }^{12}$ If candidemia is suspected, blood cultures should be taken even in the absence of fever. Consequently, treatment should be started immediately after blood cultures grow yeasts without waiting for the results of identification of Candida species and susceptibility tests. Even though the EMPIRICUS trial did not show any mortality benefit with 14-day micafungin emipirical therapy, it did decrease the rate of new fungal infections, and this empirical treatment approach is still recommended by guidelines. ${ }^{14}$ Canadian guidelines suggest that critically ill patients who meet specific criteria based on clinical prediction rules may have a benefit from empirical antifungal therapy. ${ }^{1}$

There are three main groups of antifungal agents: the azoles, the polyenes, and the echinocandins. The selection of an antifungal regimen is based on multiple factors, including, patient characteristics, epidemiological data, hospital setting, fungal strain, patient comorbidities, site of infection, and safety profiles of the antifungal agents but the most important is hemodynamic stability. Specific guidelines such as the IDSA and ESCMID guidelines are therefore available to help select optimal antifungal therapy. ${ }^{8}$ According to the current IDSA guidelines the physician can choose between fluconazole, echinocandins, amphotericin B or its lipid formulations and voriconazole (A-I). The recent guidelines of the European Society of Clinical Microbiology and Infectious Diseases (ESCMID 2011) endorse the use of echinocandins (grade A) before LipAmB (grade B) and fluconazole (grade C). Even the echinocandins are preferred when the patient has hemodynamic instability, has been previously exposed to fluconazole or when $\mathrm{C}$. glabrata is isolated. The role of amphotericin B (or its various lipid formulations) is only as an alternative drug, when there is intolerance to the other two antifungal agents, in case of refractory or resistant infections, or suspicion of infection due to non-Candida pathogens like Cryptococci. ${ }^{1}$ The duration of antifungal therapy is for two weeks, without metastatic foci of infection, after documented clearance of Candida from the bloodstream and resolution of symptoms. For those patients with metastatic foci of infection like endophthalmitis or endocarditis, a longer duration of therapy may be warranted. ${ }^{15,16}$

Overall, echinocandins are recommended as the first-line agents in most of the Candida infections, because of the following reasons: 1 . Broad spectrum activity against most of the clinically important Candida species; 2 . Better safety profile compared to other antifungal agents; and 3. Clinical superiority and fewer drug-drug interactions when compared to other systemic antifungals. In a patient-level metaanalysis of 7 clinical trials with 1,915 patients, it was observed that treatment with an echinocandin was associated with decreased mortality. ${ }^{13}$

Amongst the echinocandin class, there are subtle differences between the antifungal agents. Micafungin is non-inferior to caspofungin in the treatment of invasive candidiasis. This effect was found to be irrespective of the Candida species isolated, APACHE II score and neutropenic status at baseline as well as whether patients had candidemia or other forms of invasive candidiasis and how their catheters were managed. This non- 
inferiority to caspofungin was also demonstrated when used in treatment of esophageal candidiasis. The tolerability profile of micafungin is similar to caspofungin and better than liposomal amphotericin B. ${ }^{17}$

In patients with liver dysfunction, anidulafungin might be the best-suited echinocandin. In a study which compared the pharmacokinetics of anidulafungin on heathy volunteers versus that of patients with liver dysfunction, no significant difference was observed between the two groups. This implied that dosage adjustment is not necessary when using anidulafungin in this group of patients. Another advantage of anidulafungin is that it undergoes non-hepatic slow degradation and that it does not have any known clinically significant drug-drug interactions. ${ }^{18}$

For C. auris infections, the management consists of treatment with echinocandins (although MICs may be considerably higher compared to $C$. albicans), since they are highly resistant to fluconazole and in many cases also to voriconazole. This panel of experts recommends that owing to its high drug resistance, all $C$. auris infections should undergo antifungal sensitivity testing by default. ${ }^{16}$

\section{De-escalation}

Two weeks of antifungal treatment seem to be sufficient to prevent late complications from clinically important metastatic foci of infection in the vast majority of cases. This panel of experts was of the opinion that after treatment is initiated, blood cultures should be repeated every alternate day to document clearance of Candida from the bloodstream. In general, Treatment switching to fluconazole may be done once the patient is clinically stable, repeat blood cultures are negative and the Candida isolate is found to be sensitive to fluconazole. This strategy was shown to be safe and feasible for multiple Candida species, including C. glabrata. De-escalation to oral fluconazole may reduce exposure to echinocandins, limit the emergence of resistance to this important drug class, shorten hospital stay and limit costs. ${ }^{13}$

\section{Source control}

This panel of experts opined that central venous catheters (CVCs) are a well-documented risk factor for candidemia. $C$. albicans and $C$.parapsilosis are the most common Candida species associated with catheters and development of biofilms and are resistant to many antifungal agents. It has been seen in clinical studies that candidemia clearance is quicker if $\mathrm{CVCs}$ are removed and if they are allowed to persist, then it may be associated with higher mortality. Therefore, it was agreed upon the CVCs should be removed as early as possible if they are confirmed to be the source of infection. ${ }^{16}$ As per IDSA guidelines, nonneutropenic patients with candidemia should have a dilated ophthalmological examination, preferably performed by an ophthalmologist, within the first week after diagnosis. Chlorhexidine bathing of patients may be tried as this has been shown to reduce the rate of bloodstream infections, such as catheter related infections. ${ }^{15}$

\section{NEWER ANTIFUNGAL AGENTS}

In the last two decades, a range of antifungal agents have been developed which have proven their efficacy in management of invasive candidiasis. Currently approved echinocandins have drawbacks such as absence of an oral formulation and the need for daily administration. Therefore, to overcome these, newer agents are currently under development, and are in phase 1 or 2 trials, for the treatment and prophylaxis of invasive candidiasis, including agents with novel mechanisms of action. ${ }^{19}$

Rezafungin is a novel echinocandin currently under development, which is long-acting. It has a comparable spectrum of coverage to echinocandins and enables onceweekly extended interval dosing. It is given intravenously and has also been studied as subcutaneous administration. In-vitro studies have shown that it has potent activity against many azole-resistant and echinocandin-resistant Candida species. A phase 3 trial is currently underway to evaluate its efficacy in invasive candidiasis in comparison with caspofungin. ${ }^{19}$

SCY-078, a derivative of enfumafungin, is a semisynthetic, triterpenoid, antifungal glucan synthase inhibitor, currently in phase 3 trials for the treatment of invasive and mucocutaneous fungal diseases. It has shown good bioavailability given as once-daily administration and has been studied both as oral and intravenous. Preclinical studies have demonstrated good in-vivo activity of this molecule against both albicans and non-albicans strains of Candida. It is currently being evaluated in an open-label study for its efficacy against refractory fungal diseases. ${ }^{19}$

A new candidate drug belonging to arylamidine class, $T$ 2307 causes the collapse of mitochondrial membrane potential and is thus fungicidal. It has shown promising results both in-vitro and in-vivo against both $C$. albicans and $C$. glabrata. It is currently in phase 1 trial and is being touted as a potential antifungal agent for drug resistant Candida strains. ${ }^{19}$

\section{CONCLUSION}

Treatment of invasive candidiasis has evolved in the recent times. From the times of early-initiated risk-based empirical therapy, the therapy is shifting targeted antifungal therapy, owing to sensitive molecular diagnostic techniques. This expert panel was of the opinion that the single biggest reason for mortality in invasive candidiasis is delayed diagnosis and treatment. Hence emphasis should be given on the usage of non-culture based diagnostic techniques as well as from blood cultures, which remain the gold standard. Echinocandin class of drugs have revolutionized the management of invasive candidiasis and should be used without hesitation when the clinical situation warrants their 
usage. Among this class of antifungals, Micafungin has demonstrated non-inferiority to Caspofungin in treatment of invasive candidiasis and has demonstrated a similar tolerability profile. At the same time, older agents like fluconazole still have a place in therapy and should be used in step down therapy wherever applicable, to minimize emergence of resistance to echinocandins and preserve their antifungal efficacy. Novel antifungal agents are currently in development for the treatment options for the resistant species of Candida, such as C.glabrata and C.auris, and hopefully will lead to a better management of these resistant species in the near future. ${ }^{13}$

Funding: Funding sources from the expert group discussion was organized in association with Abbott Healthcare Pvt. Ltd.

Conflict of interest: None declared

Ethical approval: Not required

\section{REFERENCES}

1. Paramythiotou E, Frantzeskaki F, Flevari A, Armaganidis A, Dimopoulos G. Invasive fungal infections in the ICU: how to approach, how to treat. Molecules. 2014 Jan;19(1):1085-119.

2. Bajwa SJ, Kulshrestha A. Fungal infections in intensive care unit: challenges in diagnosis and management. Ann Medi Health Sci Res. 2013 Apr;3(2):238-44.

3. Lamoth F, Lockhart SR, Berkow EL, Calandra T. Changes in the epidemiological landscape of invasive candidiasis. J Antimicr Chemoth. 2018 Jan 1;73(suppl_1):i4-i13.

4. Ahmed A, Azim A, Baronia AK, Marak RS, Gurjar M. Invasive candidiasis in non-neutropenic critically illneed for region-specific management guidelines. Indian J Crit Care Med: Peer-Reviewed, Official Publication Indian Soci Crit Care Med. 2015 Jun;19(6):333-9.

5. Tsay S, Kallen A, Jackson BR, Chiller TM, Vallabhaneni S. Approach to the investigation and management of patients with Candida auris, an emerging multidrug-resistant yeast. Clini Infect Dis. 2017 Aug 17;66(2):306-11.

6. Chakrabarti A, Sood P, Rudramurthy SM, Chen S, Kaur H, Capoor M, et al. Incidence, characteristics and outcome of ICU-acquired candidemia in India. Intensive Care Med. 2015 Feb 1;41(2):285-95.

7. Morrell M, Fraser VJ, Kollef MH. Delaying the empiric treatment of Candida bloodstream infection until positive blood culture results are obtained: a potential risk factor for hospital mortality. Antimicro Agents Chemother. 2005 Sep 1;49(9):3640-5.
8. Calandra T, Roberts JA, Antonelli M, Bassetti M, Vincent JL. Diagnosis and management of invasive candidiasis in the ICU: an updated approach to an old enemy. Crit Care. 2016 Dec;20(1):125..

9. Kauffman CA. Clinical manifestations and diagnosis of candidemia and invasive candidiasis in adults. 2018. Available https://www.uptodate.com/contents/clinicalmanifestations-and-diagnosis-of-candidemia-andinvasive-candidiasis-in-adults. Accessed on 27 August 2018.

10. Clancy CJ, Nguyen MH. Diagnosing invasive candidiasis. J Clin Microbiol. 2018 May;56(5): e01909-17.

11. Eggimann P, Bille J, Marchetti O. Diagnosis of invasive candidiasis in the ICU. Ann Intens Care. 2011 Dec 1;1(1):37.

12. Badiee $\mathrm{P}$, Hashemizadeh Z. Opportunistic invasive fungal infections: diagnosis and clinical management. Indian J Medi Res. 2014 Feb;139(2):195-204.

13. Ben-Ami R. Treatment of invasive candidiasis: A narrative review. J Fungi. 2018 Sep;4(3):97.

14. Osthoff M, Khanna N, Siegemund M. The EMPIRICUS trial-the final nail in the coffin of empirical antifungal therapy in the intensive care unit?. J Thoracic Dis. 2017 Mar;9(3):E269-73.

15. Pappas PG, Kauffman CA, Andes DR, Clancy CJ, Marr KA, Ostrosky-Zeichner L, et al. Clinical practice guideline for the management of candidiasis: 2016 update by the Infectious Diseases Society of America. Clini Infect Dis. 2015 Dec 16;62(4):e1-50.

16. Kauffman CA. Management of candidemia and invasive candidiasis in adults. Available at: https://www.uptodate.com/contents/management-ofcandidemia-and-invasive-candidiasis-in-adults. Accessed on 27 August 2018.

17. Scott LJ. Micafungin: a review of its use in the prophylaxis and treatment of invasive Candida infections. Drugs. 2012 Nov 12;72(16):2141-65.

18. Yeoh SF, Lee TJ, Chew KL, Lin S, Yeo D, Setia S. Echinocandins for management of invasive candidiasis in patients with liver disease and liver transplantation. Infect Drug Resist. 2018;11:805-81.

19. Bassetti M, Righi E, Montravers P, Cornely OA. What has changed in the treatment of invasive candidiasis? A look at the past 10 years and ahead. J Antimicrob Chemother. 2018 Jan 1;73(suppl_1):i14-25.

Cite this article as: Shastri $\mathrm{P}$, Nangia V, Sathe $\mathrm{P}$, Pande R, Abraham A, Hegde A, et al. Consensus statement on the management of invasive candidiasis in Indian scenario. Int J Res Med Sci 2019;7:4410-5. 